\title{
Towards solving heterogeneous fleet vehicle routing problem with time windows and additional constraints: real use case study
}

\author{
Krzysztof Bruniecki, Andrzej Chybicki, Marek Moszyński \\ Gdańsk University of Technology, \\ Faculty of Electronics, \\ Telecommunications and Informatics, \\ ul. Narutowicza 1/2, 80-233 Gdańsk, Poland \\ Email: Krzysztof.Bruniecki@eti.pg.gda.pl
}

\author{
Mateusz Bonecki, PhD., \\ BetterSolutions \\ (BS Sp. z o. o. Sp. k.), \\ Gdańsk, Poland \\ Mateusz.Bonecki@bettersolutions.pl
}

\begin{abstract}
In advanced logistic systems, there is a need for a comprehensive optimization of the transport of goods, which would reduce costs. During past decades, several theoretical and practical approaches to solve vehicle routing problems (VRP) were proposed. The problem of optimal fleet management is often transformed to discrete optimization problem that relies on determining the most economical transport routes for a number of vehicles in order to deliver (or pick up) certain amount of goods to geographically distributed set of customers. However, real life problems generally differ from the classical cases because they impose additional constraints to be satisfied. Therefore, research related to developing dedicated theoretical and technological solutions fitted to particular real life use case are very important. In the paper, the particular variant of the VRP problem which is an exemplification of the real-life business case is presented. Authors of the paper proposed an architecture of modular system and an algorithm designed to solve the real-world variant of the VRP which is important from the business perspective.
\end{abstract}

\section{INTRODUCTION}

$\mathrm{T}$ HE fleet management support systems are becoming important tools for various branches of logistics industry. Currently, dedicated software information systems provide tools for optimal resource utilization that in consequence allows for cost reduction related to goods transportation. Therefore, recently significant number of $\mathrm{R} \& \mathrm{D}$ projects related to the problem of optimization of fleet management is being held.

In theoretical terms, the problem of fleet management is often transformed to discrete optimization problem that can be included to the class of so called "NP-hard" problems, which are generally characterized by exponential complexity. Many theoretical approaches to solve vehicle routing problem (VRP) since its first formulation by Dantzig and Ramser 1959 [1] have been studied. Heuristic approaches like Clarke and Wright algorithm [2] based on greedy routes creations or two stages approaches like "routefirst cluster-second" (or the opposite) were considered. While such heuristic approaches were mostly proposed for rather simple variants of VRP, recently, local search algorithms with metaheuristic control strategies involved

This research was sponsored by the National Centre for Research and Development within Applied Research Programme under the project "Multicriteria routing algorithms for fleet management supporting systems" (contract no. PBS3/B3/37/2015). like tabu search [3] or simulated annealing are often considered, especially for solving more complicated variants [4]. Some bio-inspired metaheuristics, including evolutionary approaches like memetic algorithms [5] or ant colony optimization techniques for VRP are also under investigation [6]. Many of the specific variants of VRP are nowadays classical. Some more prominent are Capacitated Vehicle Routing Problem (CVRP) or CVRP with time Windows (CVRPTW). However, real life transportation and logistics problems differ from such classical due to several reasons. The structure and size of real-world input datasets, like fleet heterogeneity, number of customers and many others makes them differ from classical benchmarks and therefore it forces developers to solve business scenarios independently. Also, adding additional constraints to the classical variants of VRP problems caused by business background and clients' requirements is needed. Usually, these conditions are very specific and matched to dedicated implementation. Although many approaches for VRP have been proposed so far, there is still a need to develop dedicated and fitted solutions. Therefore, research and development related VRP that arise from real-world problems is an interesting and important direction [7].

In this paper, the specific variant of VRP is considered. It includes additional constraints mostly related to time windows (TW) and truck/trailer (TT) specifics. Many variants of VRP with time windows were considered in the literature. Relatively simple variants assume only time window and capacity constraints [8]. More complex variants may add additional time dependency of travel time matrix [9] or even introduce classes of customers, e.g., pickup and delivery [10]. The truck and trailer routing problem (TTRP) were proposed and formalized by Chao [11], who also proposed 21 benchmark instances for the problem which are the classical and improved till now [12]. Even a combination of TW and TT were recently considered by Lin et al. [13] as TTRP with Time Windows (TTRPTW). However, even such complicated model is not sufficient for all the real-world demands included in business scenario considered in this article.

In the II section real-world case study was presented. It was performed on the basis of the data retrieved from the existing enterprise resource planning (ERP) system, that is actually used in leading dairy companies from northern Poland. The existing ERP system only partially supports the 
vehicle planning process for milk transport industry, so the III section presents the software architecture meant as an lightweight extension of the existing system with dedicated VRP capabilities. The proposed heuristic approach for to the specific variant of the problem and its implementation that uses constraint programming helper library (e.g., google's or-tools library) is presented in the IV section.

\section{THE PROBLEM FORMULATION AND ANALYSIS}

The considered variant of VRP can be considered as a generalization of the classical Truck and Trailer Routing Problem with Time Windows (TTRPTW), therefore it will be called GTTRPTW. Some of the additional restrictions related to GTTRPTW are vehicle heterogeneity and their possible re-use, three types of customers (i.e., not every trailer customer have to provide parking space), maximal vehicle and driver working time and distance as well as additional complexity issues for service time and transshipment time evaluation.

The time interval when the customer is serviced must be within the time window of this customer, and the type of the vehicle to service this particular customer (with or without the trailer) must be acceptable by the customer. Additionally, a list of customers at which it is possible to leave the trailer is given. The vehicle fleet consists of truck units and truck and trailer units. Trailers can be uncoupled en-route at some of the customers where truck sub-tours are built. The truck driving with a trailer can be disconnected, then pick up a load from clients, and then go back to the trailer left and go further.

The vehicle can be assigned to an additional route (or routes) if the previous route is completed. The driver may be exchanged and the vehicle limits renewed. The algorithm should exclude solutions, for which the total time of the designated vehicle route is greater than the maximum total working time of this set or situations where total route distance exceeds specified vehicle maximum route length.

Nine test cases derived from the existing ERP system were converted into GTTRPTW instances. Currently, the system works for the region of northern Poland and allows for manual route planning, that relies on assigning points to route certain vehicles, as well as automatic planning. As specified in Tab. 1, number of clients (nodes in the graph) that goods have to be transported from in 8 of 9 cases exceeds 700, except for test case no. 5. Third row represents total amount of load to be transported in particular problem instance. In this case, it is the number of liters of the milk, since the problem is specific for the dairy transport problems. Some of the node clients allow for entrance of the vehicle with the trailers and, analogically, some of the clients allow for load transshipment at their location. This information is specified in $4^{\text {th }}$ and $5^{\text {th }}$ row of the table respectively. Clients of the system also specify 3 variants of time windows constraints of load pick-up, namely: $24 \mathrm{~h}$ windows (no TW restrictions), 6:00 AM -12:00 AM (morning windows) and all-day window (6:00.AM-18:00 PM). Statistical interpretation of the input data characteristics was presented in Tab. 2. In this case, only $30 \%$ of clients allow for entrance of the trailer to their localization, and about $25 \%$ of clients allow for transshipment. Moreover, if we take under consideration fleet of available vehicles, only $10 \%-20 \%$ of units is equipped with the trailer. The considered GTTRPTW is a combination of classical VRP approach with some particular constraints related to TT and TW. However, TT only applies to $15 \%$ of vehicles of the fleet. Moreover, the detail analysis of TW constraints also shows that, for most of the clients (over $80 \%$ ) no time window is specified. For the rest of the clients, all day window (6AM-18PM) is the most often appearing.

TABLE 1.

SPECIFIED TEST CASES FOR CONSIDERED VRP PROBLEM.

\begin{tabular}{|c|c|c|c|c|c|c|c|c|c|}
\hline Use case no. & $\mathbf{1}$ & $\mathbf{2}$ & $\mathbf{3}$ & $\mathbf{4}$ & $\mathbf{5}$ & $\mathbf{6}$ & $\mathbf{7}$ & $\mathbf{8}$ & $\mathbf{9}$ \\
\hline No. of clients (nodes) & 736 & 738 & 737 & 737 & 335 & 702 & 783 & 743 & 742 \\
\hline Total load ammount & 357438 & 576772 & 456307 & 477903 & 170775 & 579107 & 622052 & 568371 & 632789 \\
\hline $\begin{array}{c}\text { The number of clients allowing for entrance } \\
\text { of a vehicle with a trailer }\end{array}$ & 218 & 234 & 240 & 212 & 98 & 212 & 237 & 218 & 231 \\
\hline No. of transhipment points & 158 & 167 & 183 & 142 & 76 & 152 & 175 & 165 & 162 \\
\hline Total no. of vehicles & 19 & 19 & 19 & 19 & 10 & 22 & 22 & 22 & 22 \\
\hline No. of vehicles with trailers & 3 & 3 & 3 & 3 & 9 & 2 & 2 & 2 & 2 \\
\hline No time window (0:00-23:59) & 596 & 585 & 596 & 585 & 266 & 702 & 783 & 743 & 742 \\
\hline Morning window 6:00 AM-12:00PM & 16 & 23 & 22 & 17 & 11 & 0 & 0 & 0 & 0 \\
\hline All-day window 6:00 AM: -18:00 PM & 124 & 130 & 119 & 135 & 58 & 0 & 0 & 0 & 0 \\
\hline
\end{tabular}


TABLE 2.

STATISTICAL OVERVIEW OF SPECIFIED TEST CASES FOR CONSIDERED VARIANT OF VRP PROBLEM.

\begin{tabular}{|c|c|c|c|c|c|c|c|c|c|}
\hline Use case no. & $\mathbf{1}$ & $\mathbf{2}$ & $\mathbf{3}$ & $\mathbf{4}$ & $\mathbf{5}$ & $\mathbf{6}$ & $\mathbf{7}$ & $\mathbf{8}$ & $\mathbf{9}$ \\
\hline $\begin{array}{c}\text { The number of customers allowing a vehicle } \\
\text { with a trailer [\%] }\end{array}$ & $30 \%$ & $32 \%$ & $33 \%$ & $29 \%$ & $29 \%$ & $30 \%$ & $30 \%$ & $29 \%$ & $31 \%$ \\
\hline No. of transhipment points [\%] & $21 \%$ & $23 \%$ & $25 \%$ & $19 \%$ & $23 \%$ & $22 \%$ & $22 \%$ & $22 \%$ & $22 \%$ \\
\hline No. of vehicles with trailers [\%] & $16 \%$ & $16 \%$ & $16 \%$ & $16 \%$ & $90 \%$ & $9 \%$ & $9 \%$ & $9 \%$ & $9 \%$ \\
\hline No time window (0:00-23:59) [\%] & $81 \%$ & $79 \%$ & $81 \%$ & $79,4 \%$ & $79,4 \%$ & $100 \%$ & $100 \%$ & $100 \%$ & $100 \%$ \\
\hline Morning window 6:00 AM-12:00PM [\%] & $2 \%$ & $3 \%$ & $3 \%$ & $2,3 \%$ & $3,3 \%$ & $0 \%$ & $0 \%$ & $0 \%$ & $0 \%$ \\
\hline All-day window 6:00 AM: -18:00 PM [\%] & $17 \%$ & $18 \%$ & $16 \%$ & $18,3 \%$ & $17,3 \%$ & $0 \%$ & $0 \%$ & $0 \%$ & $0 \%$ \\
\hline
\end{tabular}

\section{SYSTEM ARCHITECTURE}

The aim of the proposed system is to solve or validate different variants of VRP problem instances. The system may be used to find a new solution, as well as it allows to improve any previous solution when provided.

The architecture of the system, presented in Fig. 1, is modular in order to adjust to new formats and variants. The front-end layer module is handling different file formats and VRP variants for solutions and problem instances (e.g., handles proprietary JSON-based format dedicated to describe the GTTRPTW). There are also front-end modules dedicated to classical benchmarks' formats like CVRP, CVRPTW, TTRP and TTRPTW.

Data abstraction layer handles transparently different formats. It's common object-model is used by modules in the solvers' layer. The system uses different VRP solvers and the choose is up to the user. One of the solvers implemented is for GTTRPTW and is described in IV section.

The source code of the system and its modules is implemented in $\mathrm{C} / \mathrm{C}++$ language, in order to achieve high efficiency with a reasonable portability. The solvers are implemented separately and may differ from each other.

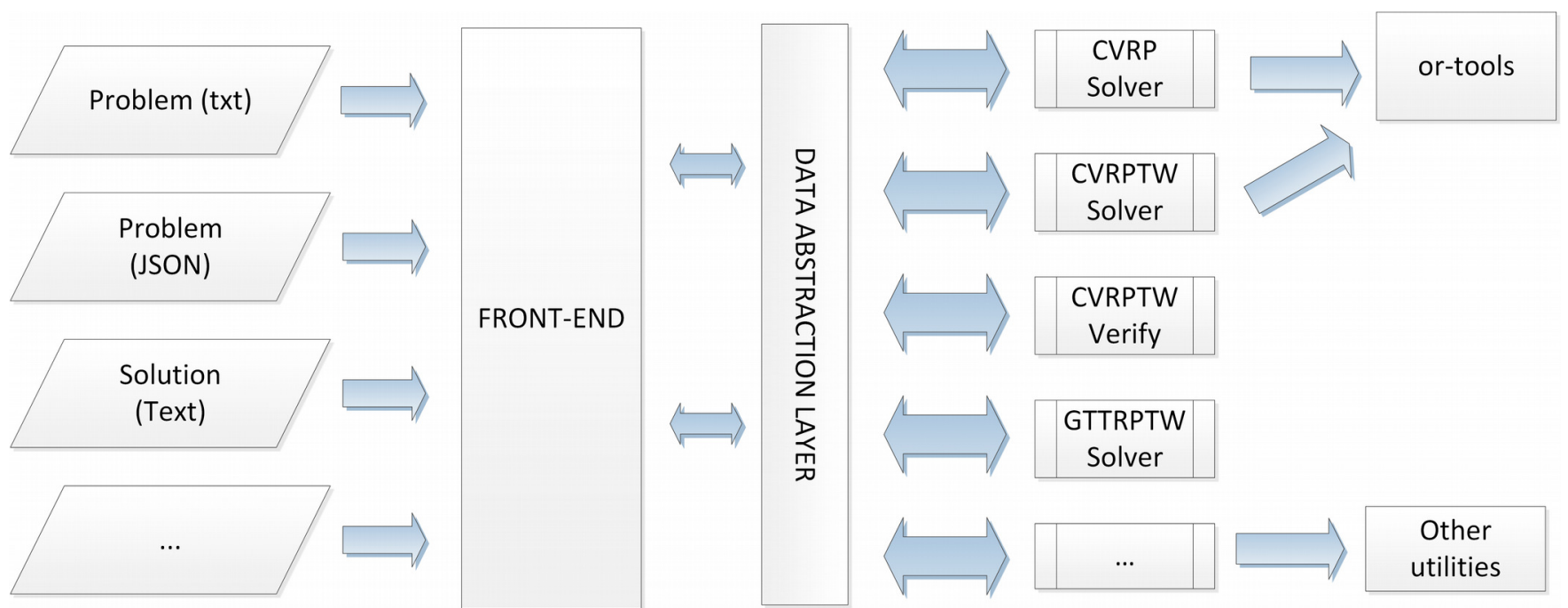

Fig. 1. The architecture of the proposed system

\section{PROPOSED APPROACH}

Already implemented solvers including GTTRPTW use constraint programming approach and third party libraries (e.g., or-tools constraint programming library). The proposed approach is based on the observation related to features of input considered test cases. Time windows in the real-world cases is not as restricting. We can distinguish between the time window related to the customer and the time window of the vehicle unit. Typical vehicle unit is often used two times during a single day (different drivers). It means that a single unit will be available within two separate time windows. Most of the customers (as can be seen in the Tab. 1 and Tab. 2) do not specify constraint on the time windows. Although, there is a significant set of customers which specify the time window, most often the time window corresponds to the single shift of the drivers. Therefore we may observe, that if the customer is served on a route by a specific truck and driver it can be often reordered within the route without violating the time window constraint. 
Therefore the outline of the strategy used in the GTTRPTW solver is as follows:

1. Find the solution $S$ of the relaxed variant (i.e., CVRPTW) of the problem;

2. For each route $r$ in the solution $S$;

if the route have truck-only customers and the vehicle is truck and trailer; we need to reorder the route and chose transhipment points;

2.1 For each transhipment customer $t$ in the route $r$ compute the route $r_{t}$;

2.1.1 Assume $t$ is the transhipment and compute the new route $r^{\prime}$ for all the customers from the original route $r$

2.1.2. Start the $r^{\prime}$ from depot to $t$ and then traverse all customers with only the truck, the number of cycles needed may vary between 1 to 3 . Finally, go back from $t$ to the depot.

2.1.2.1. The optimal cycles from $t$ are computed with use of homogeneous CVRPTW solver with $t$ considered to be the depot and the capacity of the fleet equal the capacity of the truck;

2.1.2.2. Optimize the route $r^{\prime}$ greedy by moving some truck and trailer customers from the truck cycle to the segment which represent the starting or ending part of the new route $r^{\prime}$ (the part between original depot and the assumed transhipment $t$ ).

2.1.3. Remember the route if it has smallest cost among all the previous assumptions for transhipment $t$;

2.2. Remember the best $r_{t}$ as the new route in the solution $S^{\prime}$;

3. Optimize the solution greedy by moving and exchanging some customers between routes. Must not violate other constraints.

3.1. Find a feasible move giving biggest savings,

3.1.1 If there is one go to 3.3;

3.2. Find a feasible exchange giving biggest savings

3.2.1. If there is no feasible exchange available go to 4 .

3.3. Apply the modification found

3.3.1. If the time exceeds the available then go to 4 . 
makes it its generalization - GTTRPTW. The GTTRPTW was identified during demands analysis of real-life business scenarios from dairy mil companies. Authors proposed an architecture of a lightweight extension to existing ERP system designed to solve many variants of VRP including the one proposed in this paper. Dedicated modules responsible for solving simpler variants (e.g., CVRP, CVRPTW), which are also useful in simpler business scenarios, were already implemented into the system [14]. Beside the formulation, authors presented an algorithm that solves GTTRPTW. The algorithm in its first stage uses constraint programming approach, implemented with use of or-tools library, for solving relaxed variant of problem (e.g., without TT restriction). In the second stage the algorithm continue computation with TT and additional and performs 
some local search improvements. Further research and development is planned to finally compare the results obtained by the proposed algorithm with other state of the art approaches. Since the problem variant may be considered as an extension of TTRPTW, the relaxation of the proposed algorithm and its comparison to best known results, for 36 benchmarks from [13], would be valuable. An adaptation of local search strategies like Attribute Based Hill Climbing (ABHC) into more complicated variant of TTRPTW, is also under consideration, especially that $\mathrm{ABHC}$ have revealed promising results [15] for classical TTRPTW.

\section{REFERENCES}

[1] G. B. Dantzig and J. H. Ramser "The truck dispatching problem," Management Science, vol. 6, no. 1, pp. 80-91, 1959.

[2] G. Clarke and J. Wright "Scheduling of vehicles from a central depot to a number of delivery points", Operations Research, vol. 12, no. 4, pp. 568-581, 1964.

[3] É. D. Taillard, P. Badeau, M. Gendreau, F. Guertin and J.-Y. Potvin, "A tabu search heuristic for the vehicle routing problem with soft time windows", Transportation Science, vol. 31, pp. 170-186, 1997.

[4] S.C. Ho and D. Haugland, "A tabu search heuristic for the vehicle routing problem with time windows and split deliveries", Computers \& Operations Research, vol. 31, no. 12, pp. 1947-1964, 2004.

[5] E. Osaba and F. Díaz, "Comparison of a memetic algorithm and a tabu search algorithm for the Traveling Salesman Problem," In: FedCSIS. 2012, pp. 131-136.

[6] L.M. Gambardella, E. Taillard and G. Agazzi, "MACS-VRPTW: A Multiple Ant Colony System for Vehicle Routing Problems with Time Windows" , In D. Corne, M. Dorigo and F. Glover, editors, New Ideas in Optimization McGraw-Hill, London, UK, pp. 63-76, 1999.

[7] M. Batsyn and A. Ponomarenko, "Heuristic for a Real-life Truck and Trailer Routing Problem", Procedia Computer Science vol. 31, 2014, Pages 778-792, 2nd International Conference on Information Technology and Quantitative Management, ITQM 2014.

[8] H. Akeb, A. Bouchakhchoukha, M. Hifi, "A beam search based algorithm for the capacitated vehicle routing problem with time windows". In: Computer Science and Information Systems (FedCSIS), 2013 Federated Conference on. IEEE, 2013. pp. 329-336.

[9] J. Hurkała, "Time-Dependent Traveling Salesman Problem with Multiple Time Windows" Annals of Computer Science and Information Systems, vol. 6, pp. 71-78, 2015.

[10] R. Bent and P.V. Hentenryck, "A two-stage hybrid algorithm for pickup and delivery vehicle routing problems with time windows," Computers \& Operations Research, vol.33, no.4, pp.875-893, 2003.

[11] I. M. Chao, "A tabu search method for the truck and trailer routing problem," Computers \& Operations Research, vol. 29(1), pp. 33-51, 2002.

[12] J. G. Villegasa, C. Prinsb, C. Prodhonb, A. L. Medagliac and N. Velascod, "A matheuristic for the truck and trailer routing problem", European Journal of Operational Research, vol. 230, no. 2, pp. 231-244, 2013.

[13] S.-W. Lin, V. F. Yu and C.-C. Lu, "A simulated annealing heuristic for the truck and trailer routing problem with time windows," Expert Systems with Applications, vol. 38(12), pp. 15244-15252. 2011.

[14] K. Bruniecki, A. Chybicki, M. Moszyński and M. Bonecki, "Evaluation of vehicle routing problem algorithms for transport logistics using dedicated GIS system," 2016 Baltic Geodetic Congress (BGC Geomatics), Gdansk, pp. 116-121, 2016.

[15] U. Derigs, M. Pullmann and U. Vogel, "Truck and trailer routingProblems, heuristics and computational experience," Computers \& Operations Research, vol. 40, no. 2, pp. 536-546, 2013. 